\title{
A Novel Method for Surface Defect Detection of Photovoltaic Module Based on Independent Component Analysis
}

\author{
Xuewu Zhang, Hao Sun, Yun Zhou, Ji Xi, and Min Li \\ Key Laboratory of Sensor Networks and Environmental Sensing, Hohai University, Changzhou 213022, China \\ Correspondence should be addressed to Xuewu Zhang; lab_112@126.com
}

Received 22 February 2013; Accepted 7 May 2013

Academic Editor: Vishal Bhatnagar

Copyright (c) 2013 Xuewu Zhang et al. This is an open access article distributed under the Creative Commons Attribution License, which permits unrestricted use, distribution, and reproduction in any medium, provided the original work is properly cited.

\begin{abstract}
This paper proposed a new method for surface defect detection of photovoltaic module based on independent component analysis (ICA) reconstruction algorithm. Firstly, a faultless image is used as the training image. The demixing matrix and corresponding ICs are obtained by applying the ICA in the training image. Then we reorder the ICs according to the range values and reform the de-mixing matrix. Then the reformed de-mixing matrix is used to reconstruct the defect image. The resulting image can remove the background structures and enhance the local anomalies. Experimental results have shown that the proposed method can effectively detect the presence of defects in periodically patterned surfaces.
\end{abstract}

\section{Introduction}

In recent years, developments in image processing, computer vision, artificial intelligence, and other related fields have significantly improved capability of visual inspection techniques. The objective of this paper is to explore and research effective algorithms in automatic inspection system of photovoltaic module defects.

Many products surfaces found in manufacturing have regularly patterned surfaces and can be classified as structural textures in images. The automated visual inspection in textured surfaces is then to detect small and ill-defined defects that locally break the homogeneity of a texture pattern. Numerous methods have been proposed to extract textural features either directly from spatial domain or from the spectral domain. The gray-level cooccurrence matrix (GLCM) is one of the statistic methods in the spatial domain; Zou et al. [1] present a method of Fuzzy Label Cooccurrence Matrix (FLCM) set to detect defects of colour fabric, which enhanced the computation performance in real-time application. Typically, the features in spectral domain are generally less sensitive to noise than features in spatial domain due to the relatively uniform representation in the spectral domain. Techniques in the spectral domain extract textural features by conducting frequency transforms such as Fourier transforms, Gabor transforms, or wavelet transforms. Fourier transform is a global method, which just depicts the spatial-frequency distribution without regarding to the spatial domain information [2-4]. Gabor transform belongs to windowing Fourier transform. Gabor function has well frequency description capability, which is similar to biological effects of human eye and can be used to extract the corresponding feature from different size and different direction in frequency domain. Gabor filters extract features by filtering the textured image with a set of Gabor filter banks characterized by the frequency, the sinusoid orientation, and the Gaussian function scale. However, for Gabor transform is nonorthogonal, redundancy exists in different feature components, which results in computationally intensive in analyzing texture image [5]. Recently, wavelet transform provides a convenient way to obtain a multiresolution representation, from which texture features are easily extracted. It has been a popular alternative for the extraction of textural features and has been successfully applied for texture segmentation and defect detection [6].

In recent years, independent component analysis has attracted a lot of attention in image processing applications. The basic idea in the ICA model is to construct basis images 
for a given image result in weighting components which are mutually statistically independent [7]. ICA filters are finite support filters whose size is determined by the size of the image patches selected. They are data independent in that they have been constructed from the available training data. A few studies have also been reported in the use of ICA for texture analysis. Sezer et al. [8] proposed an ICAbased defect detection method for textile fabric images. The method makes use of independent component analysis for feature extraction from the nonoverlapping subwindows of texture images. Euclidean distance between the feature obtained from average value of the feature of a defect free sample and the feature obtained from one subwindow of a test image is used to classify a subwindow as defective or nondefective. Tsai and Lai [9] proposed a fast selfcomparison scheme for defect detection in structural surfaces containing periodic complicated patterns. The scheme is simply carried out by dividing a sensed line image into two segments of equal length. An independent component analysis model is proposed to obtain the demixing matrix that can recover the translation between the two divided segments. The normalized cross-correlation is adopted to measure the similarity between two compared segments. Experimental results have shown that the proposed selfcomparison scheme can effectively and efficiently detect the presence of defects in periodically patterned surfaces. Jenssen and Eltoft $[10,11]$ presented independent component analysis of textured images as a computational technique for creating a new data dependent filter bank for use in texture segmentation.

At present, the researches mostly focus on sensory system, including scanning acoustic microscopy (SAM) [12], optical transmission [13], ultrasound lock-in thermography $[14]$, luminescence $[15,16]$, and resonance ultrasound vibration [17]. The SAM [12] method of crack assessment is not feasible for mass production of photovoltaic cells because the time required to scan a $100 \mathrm{~mm}$ by $100 \mathrm{~mm}$ wafer is between 10 and $15 \mathrm{~min}$. Additionally, the wafer has to be submerged in a water bath or covered with a water droplet. However, this approach does allow cracks as small as $5-10 \mathrm{~lm}$ to be detected. The optical crack detection system utilizes the transmission of a high intensity flashlight through the wafer and captures the image with a CCD camera coupled with optical filters [13]. To decrease the long measurement periods, infrared- (IR-) camera lifetime mapping/carrier density imaging (ILM/CDI) has been introduced recently [14]. Using an IR camera (i.e., an array of IR detectors) sensitive in the wavelength range between $\sim 3$ and $8 \mu \mathrm{m}$ instead of a single detector reduces the measurement period for a high-resolution mapping from hours to minutes and sometimes even seconds. More recently, camera-based photoluminescence imaging has been introduced, which also allows a very fast imaging of silicon wafers and solar cells at high resolution using a conventional relatively cheap silicon CCD camera $[15,16]$. Though luminescence methods are fast and non-destructive, other types of defects such as surface scratches and dislocations may interfere and misinterpret the crack identification.

This paper concentrates on research issues related to defect classification in solar cell module and discussed the general methodology as well as specific examples of the algorithms. The aim of this paper is to illustrate the potential of ICA in solar cell module defects detection. In this study, the proposed ICA-based method uses an image reconstruction strategy to eliminate the repetitive structural pattern of a solar cell surface.

The paper is organized as follows: in Section 2, firstly the basic ICA model is overviewed, and then the ICA model used for this paper is described in detail. Defect reconstruction procedure using ICA is presented in Section 3. Section 4 showed the experimental results and discussed the performance of these algorithms; Section 5 summarizes the results.

\section{Methodology}

2.1. Basic ICA Model. ICA has emerged as one powerful solution to the problem of blind source separation and has attracted broad attention.

We represent an image as a column vector $X=$ $\left[x_{1}, \ldots, x_{M}\right]^{T}$, which is modeled as linear combination of $n$ dimensional variables $s$, as shown in (1):

$$
X=\sum_{i=1}^{N} s_{i} a_{i}=A S .
$$

$S=\left[s_{1}, \ldots, s_{M}\right]^{T}$ (often described as independent components, ICs) is latent variables that cannot be directly observed, where the basis function $a_{i}, i=1, \ldots, N$, are the columns of the $(M \times N)$ matrix $A$. We usually assume $S$ is statistically independent and the mixing matrix $A$ is unknown.

In order to learn the matrix $A$, we attempt to find a linear transformation $W$ of the training data $X$, which yields a vector

$$
W X=Y,
$$

which results in components of $Y$ being as statistically independent as possible. The vector $Y$ is an estimate of $S$. Matrix $A$ is found as the pseudoinverse of $W$.

There exist several iterative algorithms performing ICA. In this research, we used FastICA algorithm proposed by Hyvarinen according to the approximation negentropy.

2.2. Preprocessing. Before applying an ICA algorithm on the images, it is usually very useful and necessary to do some preprocessing. The most basic preprocessing is to make $X$ mean zero, by subtracting $X$ by its mean. This course is called centering. Another useful preprocessing in ICA is to make each image matrix unit covariance; this course is called whitening. In this processing, $X$ is multiplied by $E D^{-1 / 2} E^{T}$, where $E$ is the orthogonal matrix of eigenvectors of $E\left(X X^{T}\right)$ and $D$ is the diagonal matrix of its eigenvalue.

\section{ICA Reconstruction Used in Defect Inspection}

The detailed procedure of the proposed scheme is summarized as follows. 
Training

(1) Select a faultless image of size $256 \times 256$ as the training image, and center and whiten the image.

(2) Compute the demixing matrix and independent components, using the fast-ICA algorithm.

(3) Sort the ICs in decreasing order and reorder the ICs.

(4) Select the number of IC and the corresponding $W_{i}$.

(5) Reform the demixing matrix $W$, and reconstruct the image $X$.

Inspection

(6) Center and whiten the inspection image $X_{I}$ of size $N \times$ $M$.

(7) Reconstruct the inspection image $X_{I}$.

(8) Binarize the reconstructed source image using the control limits:

$$
y_{i j}= \begin{cases}255 \text { (faultless point), } & \\ \mu_{i}-t \cdot \sigma_{i}<y_{i j}<\mu_{i}+t \cdot \sigma_{i}, & \text { for } i=1,2, \ldots, N ; \\ 0 \text { (defective point) }, & j=1,2, \ldots, M \\ \text { otherwise }\end{cases}
$$

where $y_{i j}$ is the gray level of image at coordinate $(i, j), \mu_{i}$ and $\sigma_{i}$ are the mean and standard deviations of the gray values in the $i$ th row of image, and $t$ is a control constant.

For each row image, $\mu_{i}-t \cdot \sigma_{i}$ and $\mu_{i}+t \cdot \sigma_{i}$ are the upper and lower control limits of intensity, respectively.

\section{Experimental Results and Analysis}

The experiments were conducted on a Pentium $41.8 \mathrm{GHz}$ personal computer processor. The FastICA package is applied to generate the demixing matrix of each training image.

4.1. Gray-Level Properties of Solar Cell Panel. A photovoltaic module is the basic element of each photovoltaic system. It consists of many jointly connected solar cells. The most commercial crystalline modules consist of 36 or 72 cells. In this paper, we have 72 cells. Every cell has size of $125 \mathrm{~mm} \times$ $125 \mathrm{~mm}$; so the width of defect should be above $1.5 \mathrm{~mm}$. Solar cells are connected and placed between a Tedlar plate on the bottom and a tempered glass on the top. Solar cells are interconnected with thin contacts on the upper side of the semiconductor material, which can be seen as a metal net on the solar cells. The net must be as thin as possible allowing a disturbance free incidence photon stream. Usually a module is framed with an aluminum frame, occasionally with a stainless steel or with a plastic frame. Figure 1 shows a faultless solar module image in a coarse resolution of $40 \times$ 967 pixels. We can see that Figure 1 has structural texture surfaces that involve only simple periodic patterns.

To further analyze the characteristics of solar cell images, we show solar cell image in fine resolution. Figures 2(a), 2(b), and 2(c) show three $2 \mathrm{D}$ images that contain a particle

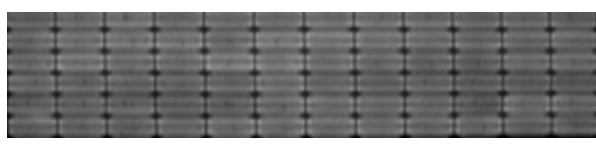

FIGURE 1: A faultless solar cell image of size $40 \times 967$ pixels.

defect in different areas of solar cell in a fine resolution. The line images in Figures 2(g)-2(i) are horizontal gray-level profiles of defective regions in Figures 2(a)-2(c) and Figures 2(d)-2(f) horizontal gray-level profiles of faultless regions corresponding to Figures 2(a)-2(c), respectively. A gray-level profile in the pixel area has the most simple and regular pattern, as seen in Figure 2(d). The pattern associated with the particle defect in one period is different to some extent from the normal one.

We can see that photovoltaic module image contains a highly repetitive pattern in a very short period.

Consider a faultless training $2 \mathrm{D}$ image as a data matrix for the ICA model. Each row vector of the matrix is treated as an observed mixture signal. Then, each row vector of the estimated source matrix obtained from the ICA model represents an independent source signal that descries a unique edge of the patterned structure in the surface. All these independent source signals compose the complete structure of a patterned solar cell panel used in training.

4.2. IC Properties of Solar Module. As shown in Figure 1, it contains 40 row images, each of size $1 \times 967$ pixels. By applying ICA to the image, 40 independent components, each of size $1 \times 967$, can be obtained. Figure 3 depicts the profiles of the resulting $40 \mathrm{ICs}$.

From Figure 3, we can see that some ICs include visible spiky points while others just have flat profiles. The ICs with spiky points can be used to represent the global structural information of a patterned solar module image. Conversely, the ICs with flat profiles can be used to represent the local uniform information of a patterned solar module image.

When using ICA for defect detection, we can first identify the proper ICs that contain significant spiky points to represent the main structure of the image. The corresponding $W_{i}$ of such identified ICs are then removed and replaced with a specific Wk that shows the flattest IC profile to reform the demixing matrix $W$. By using the reformed $W^{\prime}$ to reconstruct the inspection image, the global background texture will be removed and the local anomalies will be presented in the reconstructed image.

The ICs can be taken as basis images to represent the feature of the input image (the ICs of an image are well suited to describe the local feature variation of the image). When using a faultless patterned solar module image as an input matrix to the ICA model, the profiles of ICs will either have a flat structure or involve obvious spiky points.

4.3. ICA Reconstruction of Solar Module Image. Compute the difference between max value and min value for each IC, and then sort the ICs in decreasing order and reorder the ICs. The new ICs are shown in Table 1. 


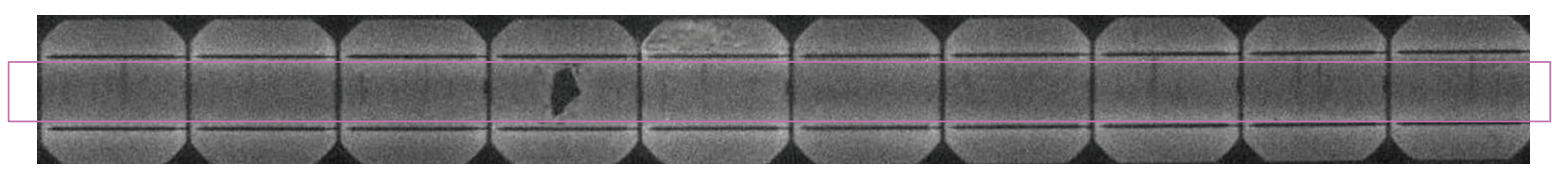

(a)

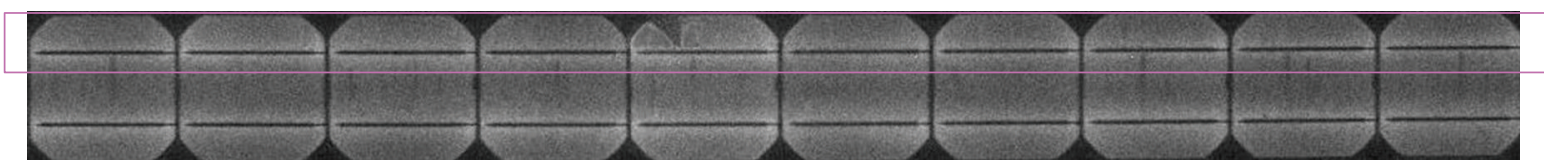

(b)

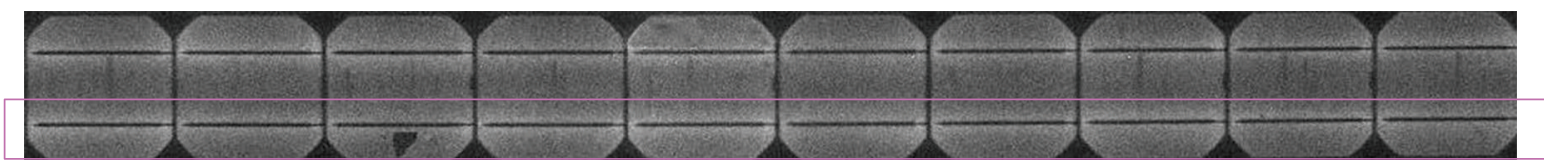

(c)

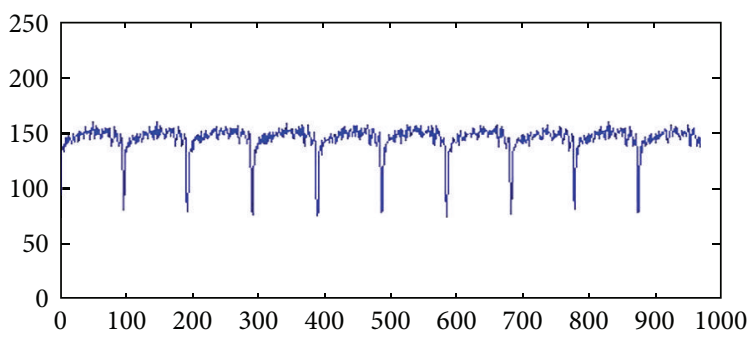

(d)

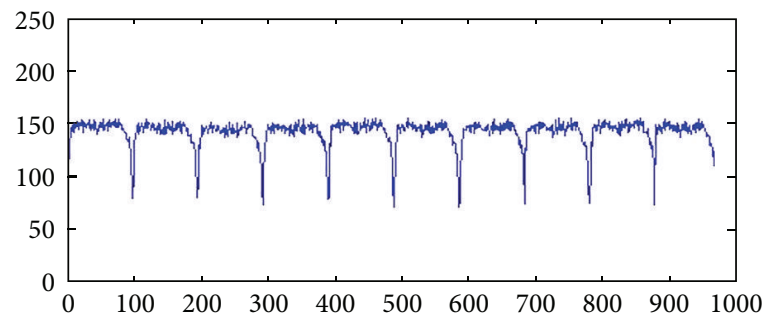

(e)

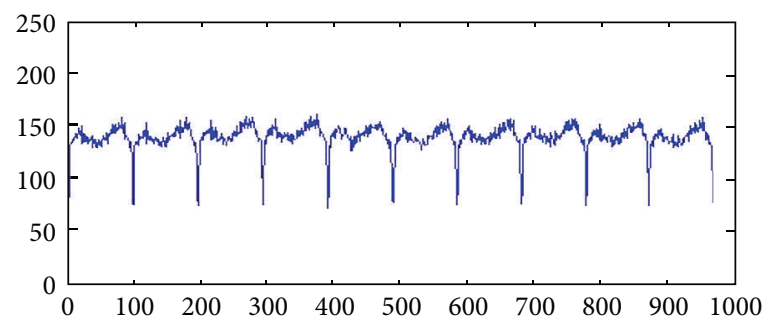

(f)

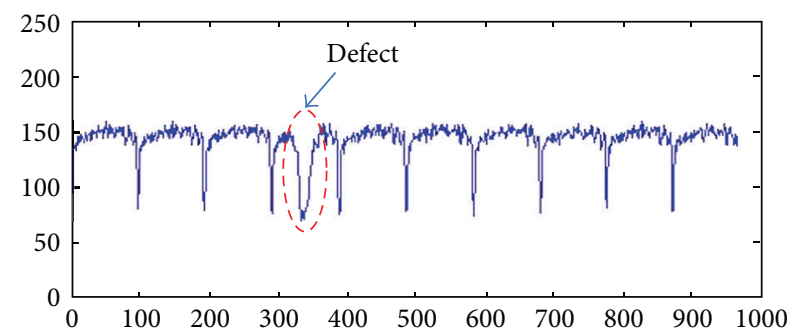

(g)

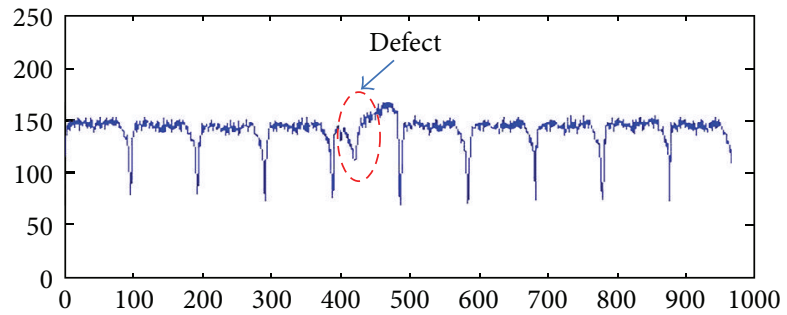

(h)

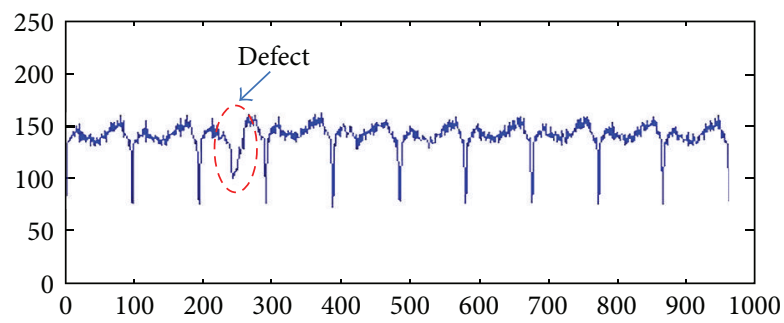

(i)

FIGURE 2: Gray profiles of solar cell panel.

Since ICA can only recover the source signals up to permutation (and scaling), the order of the 40 ICs in Figure 4 does not correspond to the row sequence in the original image $X$. It can be seen from Figure 4 that the profiles of some ICs contain visible spiky points; for example, IC2 is one of the examples. The ICs that contain obvious spiky points compose the main structural pattern of the solar cell panel image. It can also be found from Figure 3 that some ICs have near uniform profiles; for example, IC12 is one of the examples. Those ICs can be used to describe the uniform (nonstructural) background of the solar cell image.

In this paper, symmetric fixed-point ICA algorithm with $\tanh (x)$ nonlinearity is used [15].

By means of ICA, hidden factors underlying the fabric images data set are obtained. Sixteen ICs are used for the analysis. This value is obtained by trial and error. 


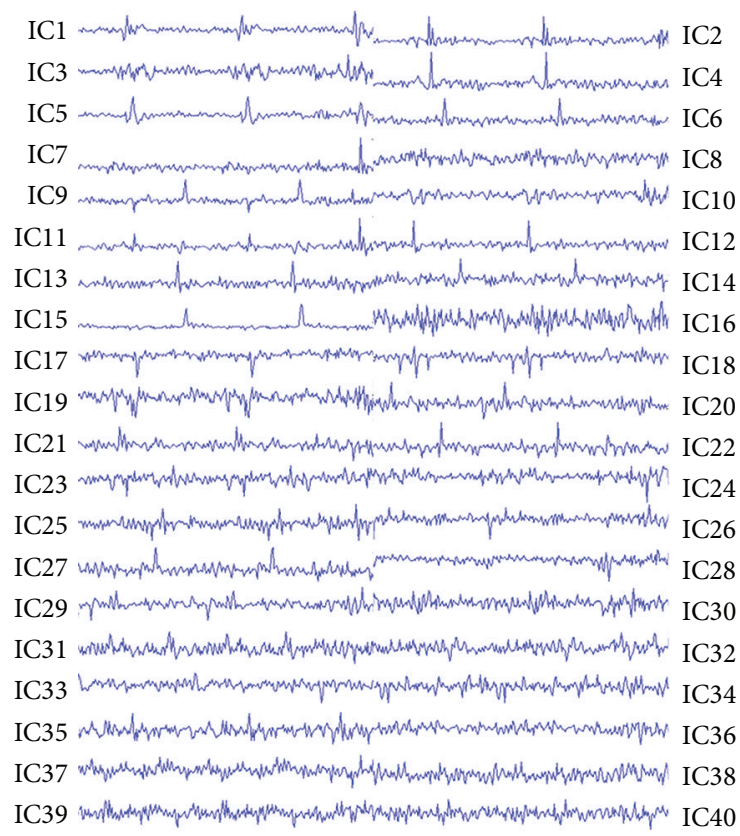

FIgURE 3: The profiles of the resulting 40 ICs.

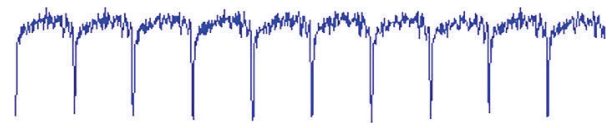

(a) Gray-level profiles

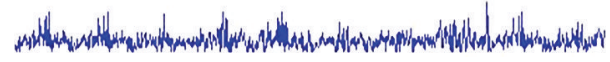

(b) $g=10$

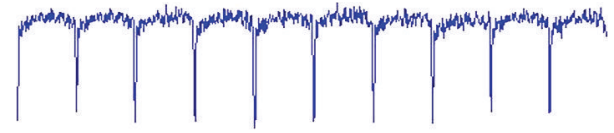

(c) $g=25$

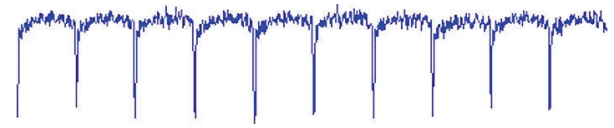

(d) $g=30$

Figure 4: Reconstruction of solar cell image using different numbers of ICs with obvious spiky points: (a) original gray-level profile of the row image in Figure 6(a); (b) (d) reconstructed row images using 10, 25, and $30 \mathrm{ICs}$, respectively.

It can be seen that IC5 has the largest range value and IC39 has the smallest range value. As mentioned in Table 1, the selection number of the replaced ICs is 25 , that is, $\mathrm{ICl}^{\prime}$, $\mathrm{IC} 2^{\prime}, \ldots, \mathrm{IC} 25^{\prime}$, and the replacing IC is IC40'. Based on the replaced ICs and the replacing $\mathrm{IC}$, the demixing matrix $W$ is reformed to a new demixing matrix $W^{*}$.

Figures 4 and 5 show the gray-level profiles of reconstruct defective image and faultless image using the new demixing matrix, respectively. It can be observed that the profile of the reconstructed row image gets closer to that of the original row
TABLE 1: ICs value.

\begin{tabular}{|c|c|}
\hline IC & $R_{i}$ \\
\hline $\mathrm{IC} 5\left(\mathrm{IC}_{1}{ }^{\prime}\right)$ & 16.2770 \\
\hline $\mathrm{ICl}\left(\mathrm{IC}_{2}{ }^{\prime}\right)$ & 16.0817 \\
\hline $\mathrm{IC10}\left(\mathrm{IC}_{3}{ }^{\prime}\right)$ & 15.5875 \\
\hline $\mathrm{IC} 3\left(\mathrm{IC}_{4}{ }^{\prime}\right)$ & 14.3378 \\
\hline $\mathrm{IC} 2\left(\mathrm{IC}_{5}{ }^{\prime}\right)$ & 14.0981 \\
\hline $\operatorname{IC} 8\left(\mathrm{IC}_{6}{ }^{\prime}\right)$ & 12.9055 \\
\hline $\operatorname{IC} 11\left(\mathrm{IC}_{7}{ }^{\prime}\right)$ & 12.8781 \\
\hline $\mathrm{IC} 16\left(\mathrm{IC}_{8}{ }^{\prime}\right)$ & 12.7694 \\
\hline $\mathrm{IC} 7\left(\mathrm{IC}_{9}{ }^{\prime}\right)$ & 12.4899 \\
\hline $\mathrm{IC} 4\left(\mathrm{IC}_{10}{ }^{\prime}\right)$ & 11.9651 \\
\hline $\operatorname{IC} 23\left(\mathrm{IC}_{11}{ }^{\prime}\right)$ & 11.2746 \\
\hline $\operatorname{IC} 20\left(\mathrm{IC}_{12}{ }^{\prime}\right)$ & 10.7203 \\
\hline $\operatorname{IC9}\left(\mathrm{IC}_{13}{ }^{\prime}\right)$ & 10.6168 \\
\hline $\operatorname{IC12}\left(\mathrm{IC}_{14}{ }^{\prime}\right)$ & 10.6159 \\
\hline $\operatorname{IC} 21\left(\mathrm{IC}_{15}{ }^{\prime}\right)$ & 10.4436 \\
\hline $\operatorname{IC17}\left(\mathrm{IC}_{16}{ }^{\prime}\right)$ & 10.1953 \\
\hline IC6 $\left(\mathrm{IC}_{17}{ }^{\prime}\right)$ & 10.0291 \\
\hline $\operatorname{IC} 25\left(\mathrm{IC}_{18}{ }^{\prime}\right)$ & 9.9173 \\
\hline $\operatorname{IC19}\left(\mathrm{IC}_{19}{ }^{\prime}\right)$ & 9.5628 \\
\hline $\mathrm{IC} 24\left(\mathrm{IC}_{20}{ }^{\prime}\right)$ & 9.4935 \\
\hline $\mathrm{IC} 22\left(\mathrm{IC}_{21}{ }^{\prime}\right)$ & 9.4757 \\
\hline $\operatorname{IC13}\left(\mathrm{IC}_{22}{ }^{\prime}\right)$ & 9.3286 \\
\hline $\operatorname{IC} 28\left(\mathrm{IC}_{23}{ }^{\prime}\right)$ & 9.1619 \\
\hline $\mathrm{IC} 14\left(\mathrm{IC}_{24}{ }^{\prime}\right)$ & 9.0582 \\
\hline $\operatorname{IC} 30\left(\mathrm{IC}_{25}{ }^{\prime}\right)$ & 8.8652 \\
\hline $\operatorname{IC} 29\left(\mathrm{IC}_{26}{ }^{\prime}\right)$ & 8.8194 \\
\hline $\operatorname{IC} 32\left(\mathrm{IC}_{27}{ }^{\prime}\right)$ & 8.7825 \\
\hline $\operatorname{IC} 26\left(\mathrm{IC}_{28}{ }^{\prime}\right)$ & 8.7541 \\
\hline $\mathrm{IC} 33\left(\mathrm{IC}_{29}{ }^{\prime}\right)$ & 8.5220 \\
\hline $\operatorname{IC} 31\left(\mathrm{IC}_{30}{ }^{\prime}\right)$ & 8.4803 \\
\hline $\operatorname{IC} 35\left(\mathrm{IC}_{31}{ }^{\prime}\right)$ & 8.4722 \\
\hline $\operatorname{IC} 36\left(\mathrm{IC}_{32}{ }^{\prime}\right)$ & 8.4665 \\
\hline $\mathrm{IC} 18\left(\mathrm{IC}_{33}{ }^{\prime}\right)$ & 8.4331 \\
\hline $\mathrm{IC} 27\left(\mathrm{IC}_{34}{ }^{\prime}\right)$ & 8.2911 \\
\hline $\mathrm{IC} 40\left(\mathrm{IC}_{35}{ }^{\prime}\right)$ & 8.1047 \\
\hline $\mathrm{IC} 38\left(\mathrm{IC}_{36}{ }^{\prime}\right)$ & 8.1013 \\
\hline $\operatorname{IC} 34\left(\mathrm{IC}_{37}{ }^{\prime}\right)$ & 8.0307 \\
\hline $\operatorname{IC} 15\left(\mathrm{IC}_{38}{ }^{\prime}\right)$ & 7.9053 \\
\hline IC39 $\left(\mathrm{IC}_{39}{ }^{\prime}\right)$ & 7.8023 \\
\hline $\operatorname{IC} 37\left(\mathrm{IC}_{40}{ }^{\prime}\right)$ & 7.2717 \\
\hline
\end{tabular}

image as the number of spiky ICs is increased. Note that 25 out of a total of 40 ICs are enough to reconstruct the main structure of the original row image, as shown in Figures 4 and 5.

Figure 6 shows the typical samples of solar cell module. Figure 7 shows the reconstructed images by using the newly 


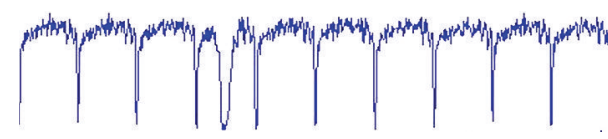

(a) Gray-level profiles

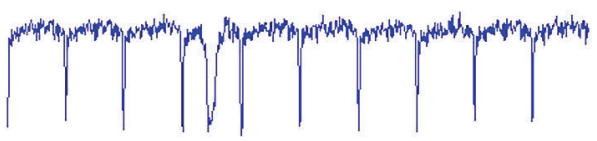

(c) $g=25$

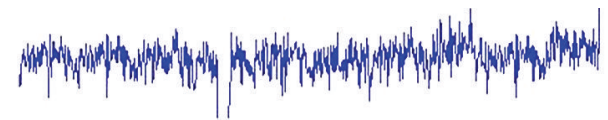

(b) $g=10$

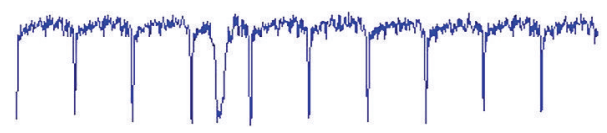

(d) $g=30$

FIGURE 5: Reconstruction of solar cell image using different numbers of ICs with obvious spiky points: (a) original gray-level profile of the row image in Figure 5(a); (b) (d) reconstructed row images using 10, 25, and 30 ICs, respectively.

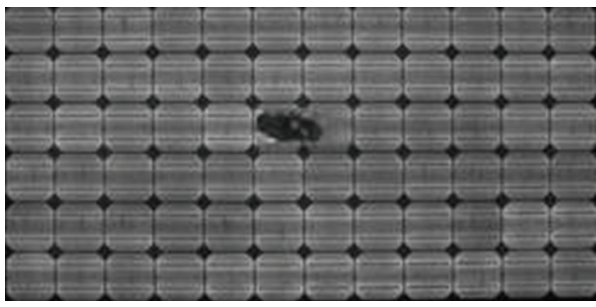

(a) Hole

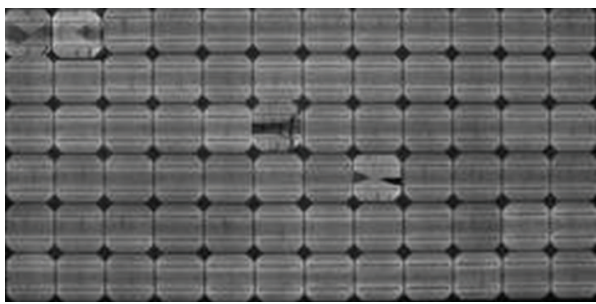

(c) Crack

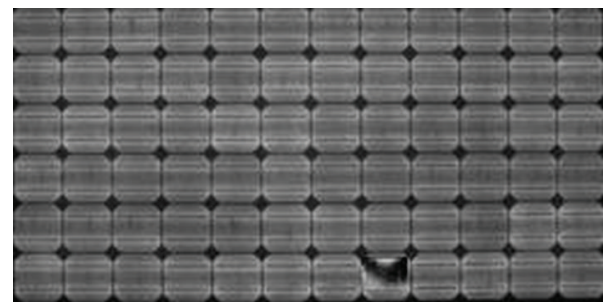

(b) Sintering

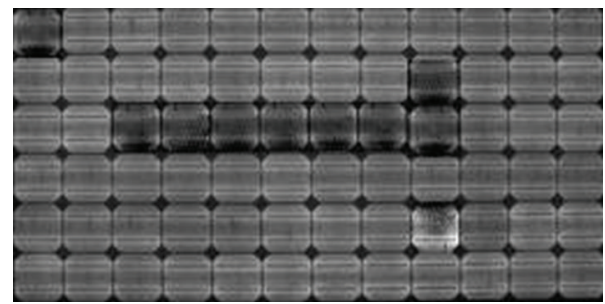

(d) Cobwebbing

FIgURE 6: Typical samples.

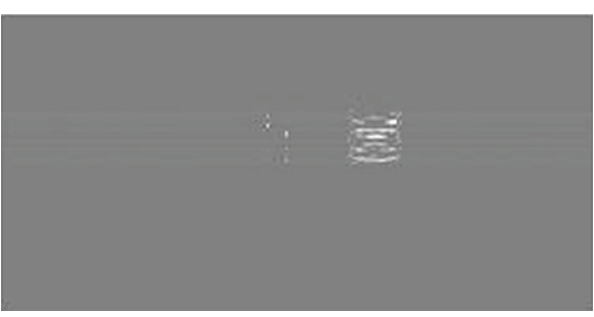

(a) Hole

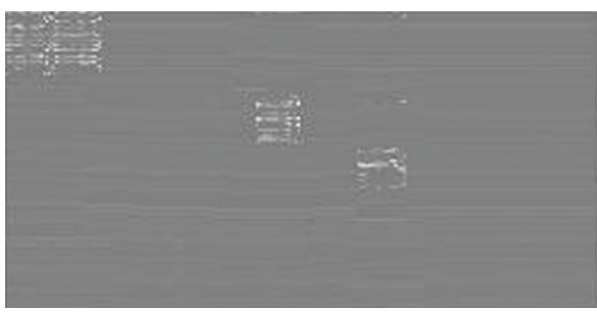

(c) Crack

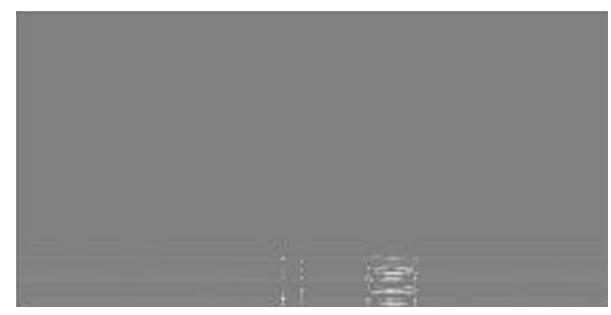

(b) Sintering

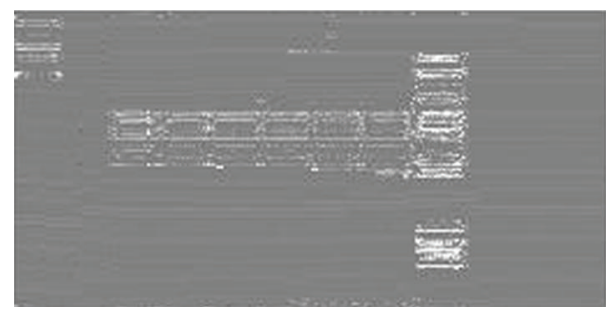

(d) Cobwebbing

FIGURE 7: Reconstruction results using ICA. 


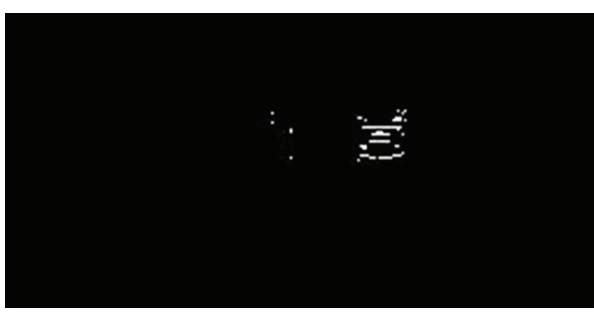

(a) Hole

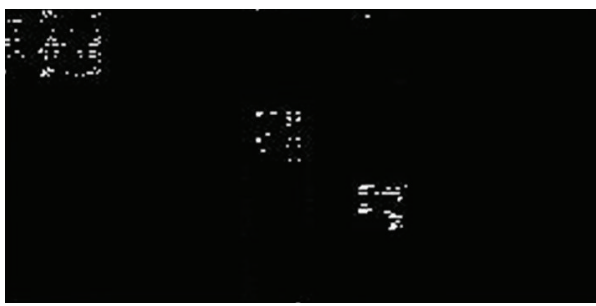

(c) Crack

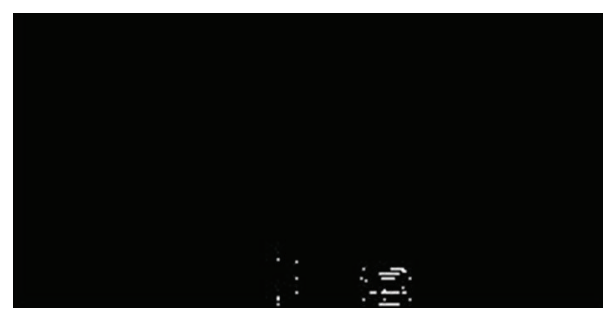

(b) Sintering

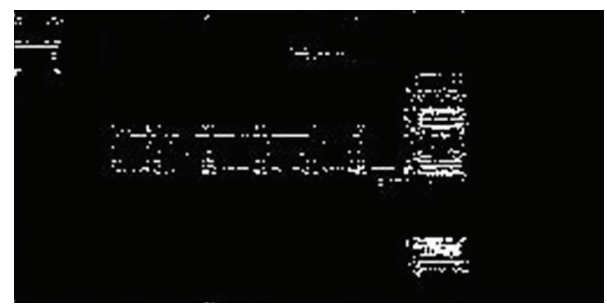

(d) Cobwebbing

Figure 8: Binary results.

reformed demixing matrix to reconstruct the test images in Figure 6, and Figure 8 shows the binary results. We can see that, for defective images, the background texture is effectively eliminated and defects are well segmented. For the faultless image, the resulting binary image is uniformly white and no defect is claimed.

If the gray value $k$ falls with the control limits, it is classified as a faultless point of the inspection surface. Otherwise, it is classified as a defective point. The constant $t$ is taken by 2.5 here.

\section{Conclusions}

In this study, an ICA-based system for inspection of photovoltaic module defects was developed. A photovoltaic module consists of many jointly connected solar cells, so it can be processed as repetitive patterns. A faultless image is used as the training image. The demixing matrix and corresponding ICs are obtained by applying the ICA in the training image. Then we re-order the ICs according to the range values and reform the demixing matrix. Then the reformed demixing matrix is used to reconstruct the defect image. The resulting image can remove the background structures and enhance the local anomalies. Experimental results have shown that the proposed method can effectively detect the presence of defects in periodically patterned surfaces. This method correctly locates the position of defects but does not reflect the true shape of the defects, and the length of defect size is above $1.5 \mathrm{~mm}$.

\section{Acknowledgments}

This paper is supported by the National Natural Sciences Foundation of China (Grant no. 61273170); Ph.D. Programs Foundation of the Ministry of Education of China
(20120094120023); Changzhou Science and technology Support Program (Industrial) (no. CE20120101). Xuewu Zhang is an Associate Professor of communication engineering at the College of Computer and Information, Hohai University. He received the B.S. degree, the M.S. degree, and the Ph.D. degree from the Computer and Information College, Hohai University in 1998, 2006, and 2011, respectively. He has published over 40 research papers and obtained over 10 patents. His research interests include pattern recognition, multimedia signal processing, machine learning, and bionics.

\section{References}

[1] C. Zou, B. W. Wang, and Z. G. Sun, "Detection of fabric defects with Fuzzy Label Co-occurrence Matrix set," Journal of Donghua University, vol. 26, no. 5, pp. 549-553, 2009.

[2] H. Y. T. Ngan, G. K. H. Pang, S. P. Yung, and M. K. Ng, "Wavelet based methods on patterned fabric defect detection," Pattern Recognition, vol. 38, no. 4, pp. 559-576, 2005.

[3] X. W. Zhang, L. Z. Xu, Y. Q. Ding, X. N. Fan, L. P. Gu, and H. Sun, "Inspection of surface defects in copper strip based on machine vision," Life System Modeling and Intelligent Computing, vol. 6328, no. 1, pp. 304-312, 2010.

[4] S. C. Kim and T. J. Kang, "Texture classification and segmentation using wavelet packet frame and Gaussian mixture model," Pattern Recognition, vol. 40, no. 4, pp. 1207-1221, 2007.

[5] O. Pichler, A. Teuner, and B. J. Hosticka, "A comparison of texture feature extraction using adaptive Gabor filtering, pyramidal and tree structured wavelet transforms," Pattern Recognition, vol. 29, no. 5, pp. 733-742, 1996.

[6] T. S. Li, "Applying wavelets transform and support vector machine for copper clad laminate defects classification," Computers and Industrial Engineering, vol. 56, no. 3, pp. 1154-1168, 2009.

[7] A. Hyvärinen and E. Oja, "Independent component analysis: algorithms and applications," Neural Networks, vol. 13, no. 4-5, pp. 411-430, 2000. 
[8] O. G. Sezer, A. Ertuzun, and A. Ercil, "Independent component analysis for texture defect detection," Pattern Recognition and Image Analysis, vol. 14, no. 2, pp. 303-307, 2004.

[9] D. M. Tsai and S. C. Lai, "Defect detection in periodically patterned surfaces using independent component analysis," Pattern Recognition, vol. 41, no. 9, pp. 2812-2832, 2008.

[10] R. Jenssen and T. Eltoft, "ICA filter bank for segmentation of textured images," in Proceedings of the 4th International Symposium on Independent Component Analysis and Blind Signal Separation (ICA '03), Nara, Japan, 2003.

[11] R. Jenssen and T. Eltoft, "Independent component analysis for texture segmentation," Pattern Recognition, vol. 36, no. 10, pp. 2301-2315, 2003.

[12] A. Belyaev, O. Polupan, S. Ostapenko, D. Hess, and J. P. Kalejs, "Resonance ultrasonic vibration diagnostics of elastic stress in full-size silicon wafers," Semiconductor Science and Technology, vol. 21, no. 3, pp. 254-260, 2006.

[13] E. Rueland, A. Herguth, A. Trummer, S. Wansleben, and P. Fath, "l-Crack detection and other optical characterization techniques for in-line inspection of wafers an cells," in Proceedings of 20th EU PVSEC, pp. 3242-3245, Barcelona, Spain, 2005.

[14] J. P. Rakotoniaina, O. Breitenstein, M. H. Al Rifai, D. Franke, and A. Schnieder, "Detection of cracks in silicon wafers and solar cells by lock-in ultrasound thermography," in Proceedings of $P V$ Solar conference, pp. 640-643, Paris, France, 2004.

[15] T. Trupke, R. A. Bardos, M. C. Schubert, and W. Warta, "Photoluminescence imaging of silicon wafers," Applied Physics Letters, vol. 89, no. 4, Article ID 044107, 2006.

[16] T. Fuyuki, H. Kondo, T. Yamazaki, Y. Takahashi, and Y. Uraoka, "Photographic surveying of minority carrier diffusion length in polycrystalline silicon solar cells by electroluminescence," Applied Physics Letters, vol. 86, no. 26, Article ID 262108, pp. $1-3,2005$.

[17] A. Belyaev, O. Polupan, W. Dallas, S. Ostapenko, D. Hess, and J. Wohlgemuth, "Crack detection and analyses using resonance ultrasonic vibrations in full-size crystalline silicon wafers," Applied Physics Letters, vol. 88, no. 11, Article ID 111907, 2006. 


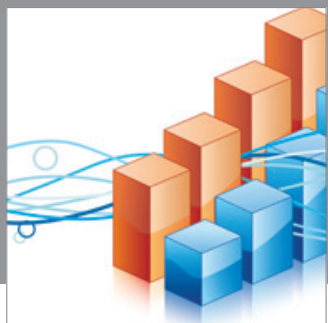

Advances in

Operations Research

mansans

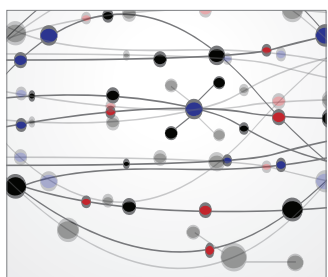

The Scientific World Journal
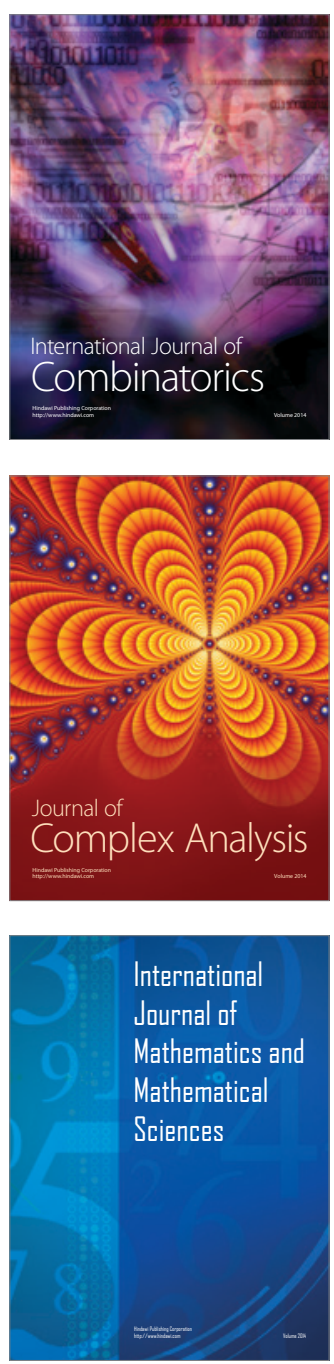
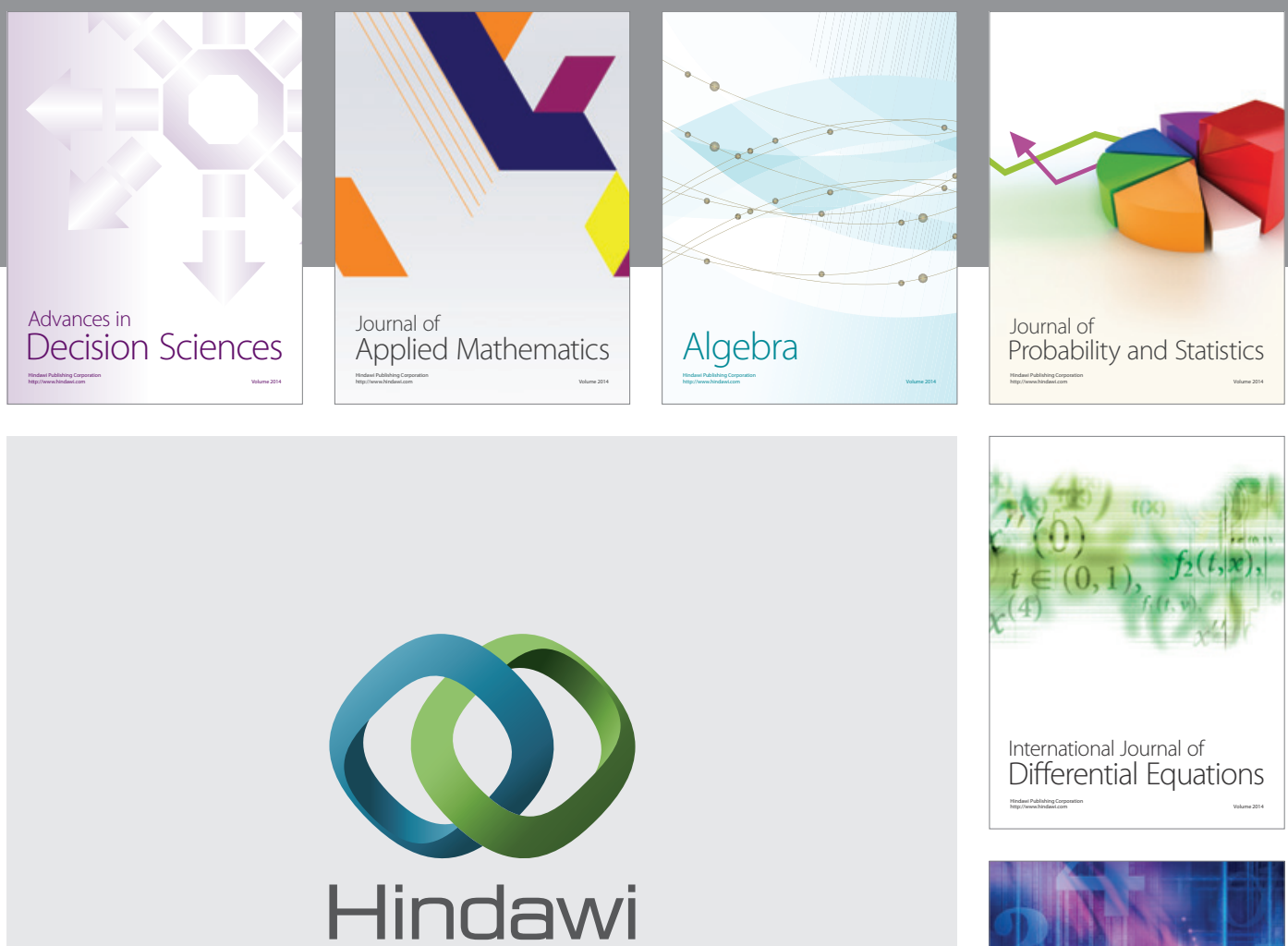

Submit your manuscripts at http://www.hindawi.com
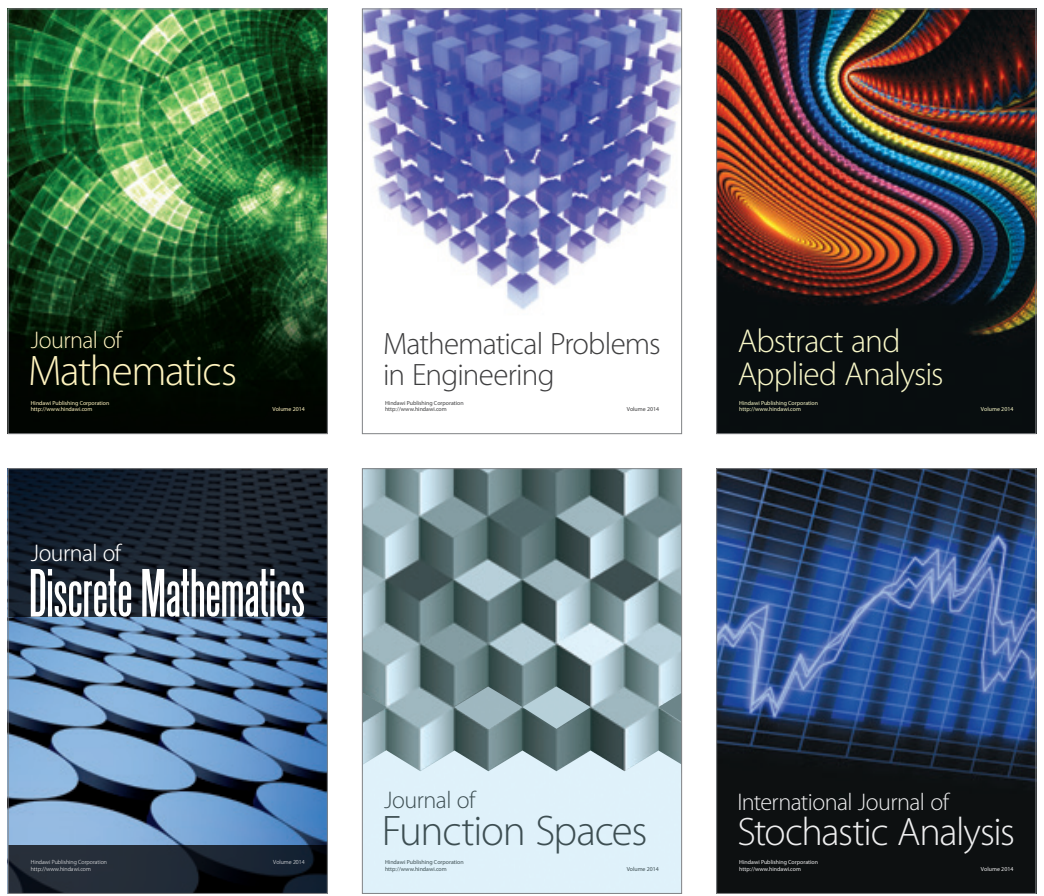

Journal of

Function Spaces

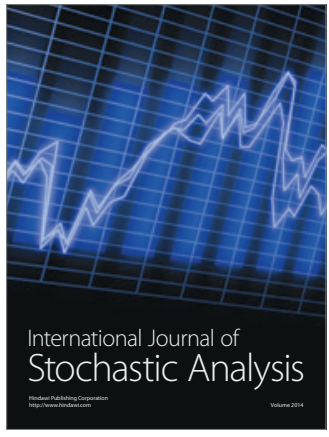

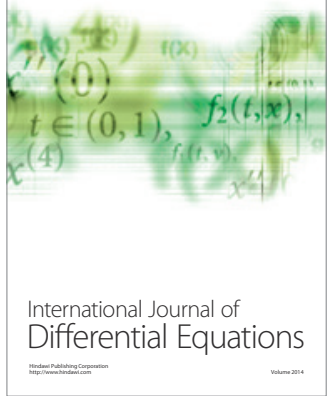
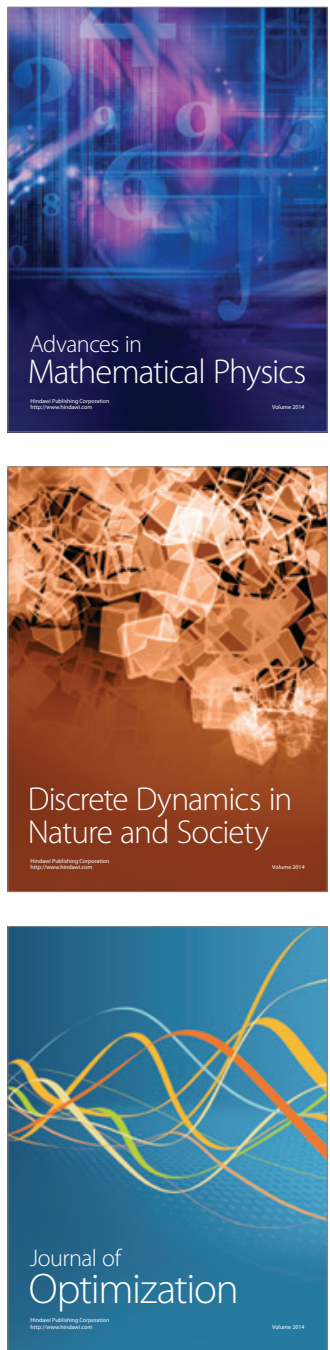